\title{
Comparisons of tumor-infiltrating lymphocyte levels and the 21-gene recurrence score in ER-positive /HER2-negative breast cancer
}

\author{
Sung Gwe Ahn ${ }^{1+}$, Yoon Jin Cha ${ }^{2+}$, Soon June Bae ${ }^{1}$, Chanik Yoon ${ }^{1}$, Hak Woo Lee ${ }^{1}$ and Joon Jeong ${ }^{1 *}$ (D)
}

\begin{abstract}
Background: Recent studies have shown that tumors with extensive tumor-infiltrating lymphocytes (TILs) have a higher probability of pathologic complete response, even in luminal/human epidermal growth factor 2 (HER2)negative breast cancer. We compared TIL levels and the 21-gene recurrence score (RS) in estrogen receptor (ER)positive/HER2-negative breast cancer.
\end{abstract}

Methods: We evaluated the percentage of stromal TILs in 198 ER-positive/HER2-negative patients in whom RS was obtained by examining slides of surgical specimens by standardized methodology proposed by the international TIL Working Group. TIL levels were categorized as high ( $\geq 60 \%)$, intermediate (11-59\%), or low ( $\leq 10 \%)$. All tumors were treatment-naïve.

Results: Ninety-seven (49.0\%), 88 (44.4\%), and 13 patients (6.6\%) had low, intermediate, and high TIL levels, respectively. There was a significant but weak correlation between continuous RS and continuous TIL levels (Pearson's $R=0.201, p=0$. 004). The mean RS was significantly highest in high TIL tumors (17.8 \pm 10.7 in low TIL tumors, $19.4 \pm 8.7$ in intermediate TIL tumors, and $26.2 \pm 8.2$ in high TIL tumors; $p=0.014$ ). However, when we compared categorized RS and TIL levels, we found that tumors with high TIL levels tended to have higher RS $(\geq 26)$ but it was not significant $(p=0.155)$. Furthermore, multivariate analysis revealed that high RS was not an independent factor associated with high TIL levels. Chemo-endocrine therapy was more frequently performed among patients with high TILs and less frequently among those with low or intermediate TILS $(p<0.001)$.

Conclusions: Despite of a weak correlation between continuous TIL levels and RS, we found that tumors with high TIL levels tended to have a higher RS in ER-positive/HER2-negative breast cancer. Further study is warranted considering the clinical outcomes.

Keywords: Tumor-infiltrating lymphocytes, 21-gene recurrence score, Breast cancer

\section{Background}

The presence of tumor-infiltrating lymphocytes (TILs) reflects an adaptive anti-tumor immune response [1]. Although the immunogenicity of breast cancer has not been strongly considered in research or clinical practice, TILs are emerging as biomarkers for predicting clinical response to

\footnotetext{
* Correspondence: gsjjoon@yuhs.ac

${ }^{\dagger}$ Equal contributors

${ }^{1}$ Department of Surgery, Gangnam Severance Hospital, Yonsei University College of Medicine, Seoul, Korea

Full list of author information is available at the end of the article
}

chemotherapy for breast cancer [2-8]. Recent translational studies from large prospective trials suggest that tumors with high TIL levels show a superior prognosis compared to tumors with low TIL levels in breast cancer patients treated with neoadjuvant or adjuvant chemotherapy [2-8]. The correlation between high TIL levels and improved clinical outcome was most prominent in triple-negative breast cancer (TNBC) [2, 6-8]. After the initial report from the BIG2-98 trial, a positive relationship between TIL levels and clinical outcome in TNBC was validated in independent cohorts in two clinical trials $[2,6]$. 
Interestingly, TIL levels have not been shown to be prognostic in patients with estrogen receptor (ER)-positive tumors receiving adjuvant chemotherapy. However, a recent study reported that patients with a high level of TILs have a greater chance of obtaining a pathological complete response (pCR), even in hormone receptor (HR)-positive/human epidermal growth factor receptor 2 (HER2)-negative breast cancers [9]. In addition, a recent meta-analysis showed that HR-positive/HER2-negative tumors with high TIL levels at baseline biopsy, prior to chemotherapy, have a higher probability of pCR $(n=1366)$ [10].

The 21-gene recurrence score (RS) is derived from a polymerase chain reaction-based test that examines 16 tumor related genes and 5 reference genes to analyze cell proliferation, estrogen signaling, and invasion capacity. It has been shown to predict the clinical benefit of chemotherapy for individuals with ER-positive/HER2negative breast cancer [11, 12]. Furthermore, previous studies have shown that tumors with a high RS have a higher rate of $\mathrm{pCR}$ in the neoadjuvant setting $[13,14]$.

Both TIL levels and the RS could serve as biomarkers associated with chemotherapy responsiveness in HR-positive/ HER2-negative breast cancer. Moreover, identifying suitable patients among those with HR-positive/HER2-negative cancers who derive clinical benefit from chemotherapy has been a critical issue; however, the relationship between the two markers has not been intensively examined.

In this study, we examined the association between TIL levels and RS in patients with ER-positive/HER2negative breast cancer who did not receive neoadjuvant chemotherapy (NAC).

\section{Methods}

\section{Patients}

Between August 2011 and April 2017, eligible patients were identified from the breast cancer database of the Gangnam Severance Hospital [15]. During the period, 212 patients underwent Oncotype DX testing. All patients had ERpositive/HER2-negative breast cancer and did not undergo NAC. In 198 patients, TIL levels were successfully evaluated using treatment-naïve surgical specimens. A pathologist (YJC) reviewed the histologic features by using Hematoxylin \& eosin (H\&E)-stained slides for all cases. Histologic grade for breast cancer was assessed using the Nottingham grading system [16]. The institutional review board of the Gangnam Severance Hospital, approved the study to be in accordance with guidelines of good clinical practice and the Declaration of Helsinki. The need for informed consent was waived under the approval of the institutional review board due to the retrospective design.

\section{Assessment of TIL levels}

Stromal TIL levels were scored according to the guidelines for TIL assessment by the TILs Working Group
[1]. H\&E-stained whole tumor slides were examined and scored by a pathologist (YJC). Briefly, the tumor area as defined by the presence of invasive tumor was evaluated, and all mononuclear cells, including lymphocytes and plasma cells but not polymorphonuclear leukocytes, were scored. Areas outside of the tumor border, around the intraductal component, and normal lobules were excluded. Within the tumor border, TILs with crush artifacts and necrosis were excluded. For each case, three representative tumor areas were evaluated for TILs, and the average score was reported as a percentage. TIL levels was categorized as high $(\geq 60 \%)$, intermediate (11-59\%), or low ( $\geq 10 \%)$. The cut-off point was defined using the criteria suggested by Denkert et al. [10].

\section{Immunohistochemistry}

For immunohistochemistry (IHC), antibodies specific for ER (1:100 clone 6F11; Novocastra, Newcastle upon Tyne, UK), progesterone receptor (PR; clone 16; Novocastra), HER2 (4B5 rabbit monoclonal antibody; Ventana Medical Systems, Tucson, AZ, USA), and Ki-67 (MIB-1; Dako, Glostrup, Denmark) were stained using formalinfixed, paraffin-embedded tissue sections [17]. Positivity of ER and PR IHC expression was defined according to the modified Allred system: positive, Allred score 2-8; and negative, Allred score 0-1 [18]. All tumors included in this study had ER positivity (Allred scores $\geq 3$ ). HER2 status was considered positive with a score of $3+$ and negative with a score of 0 or $1+$ [19]. Tumors with a score of $2+$ underwent fluorescent in situ hybridization analysis according to the manufacturer's instructions (PathVysion kit; Vysis, Downers Grove, IL, USA or HER2 inform; Ventana) [19]. Ki-67 expression was evaluated by $\mathrm{YJC}$ and displayed presented as a percentage (range $0-100 \%$ ) of positive tumor cells.

\section{Oncotype $\mathrm{dx}$ assays}

The RS is calculated by the Oncotype Dx assay $[11,12]$. RS scores are calculated on a scale from 0 to 100 and derived from the reference-normalized expression measurements of 16 cancer-related genes (Ki67, STK15, Survivin or BIRC5, CCNB1 or cyclin B1, MYBL2, GRB7, HER2, ER, PGR, BCL2, SCUBE2, MMP11 or stromelysin 3, CTSL2 or cathepsin L2, GSTM1, CD68, and BAG1) and five reference genes. Quantitative single gene scores are determined via reverse transcriptase-polymerase chain reaction. Expression of each gene was measured in triplicate and normalized to a set of five reference genes (beta-actin [ACTB], GAPDH, GUS, RPLPO, and TFRC). Reference-normalized expression measurements ranged from 0 to 15, where a one-unit increase approximately reflects a two-fold increase in RNA.

Tumors were classified into the following categories: low risk $(\mathrm{RS}<18)$, intermediate risk ( $\mathrm{RS} 18-30)$, and high risk 
$(\mathrm{RS} \geq 31)$. The RS was also dichotomized with a cutoff of 26 as in the TAILORX trial [20]. The Oncotype Dx assay was supplied by Genomic Health (Redwood City, CA, USA) and performed using RNA extracted from formalinfixed paraffin-embedded tissue. After a review of hematoxylin and eosin-stained slides to determine whether sufficient invasive breast cancer was present and whether manual microdissection was indicated, RNA was extracted from the unstained sections. Cases without tumor samples (i.e., depleted by prior tissue studies) or with cancer cells occupying $<5 \%$ of the section area were excluded from the assay [11]. All tissues from patients in this study were successfully analyzed.

\section{Statistical analysis}

The primary objective of this study was to test the correlation between RS and TIL levels. Pearson's R was calculated to measure the correlative value between two continuous scores. Discrete variables were compared using the $\mathrm{X} 2$ test. Continuous variables were compared using the Mann-Whitney U and Kruskal-Wallis tests. Student's t-test or one-way analysis of variation (ANOVA) was used to compare means. The variables with statistical significance in the univariate analysis were included in the selection of the full multivariate model, and backward elimination was taken to arrive at the final model.

The Kolmogorov-Smirnov test was applied to test the normal distribution of continuous variables, including RS and TIL levels. The distributions of nonparametric variables were compared using the Kruskal-Wallis test. A post-hoc test was performed using the Bonferronicorrected Dunn's procedure. SPSS version 18 (SPSS Inc., Chicago, IL, USA) was used to perform the statistical analyses. Statistical significance was defined as a $p$-value $<0.05$.

\section{Results}

\section{Baseline characteristics}

One hundred ninety-eight patients with ER-positive/HER2negative tumors were included in the analyses. Ninety-eight (50\%), $80(40 \%)$, and 20 patients $(10 \%)$ had a low, intermediate, and high RS, respectively, while $97(49 \%), 88$ (44\%), and 13 patients (7\%) had low, intermediate, and high TIL levels, respectively. The baseline characteristics are presented in Table 1. The median age of these patients was 49 years (range: $27-75$ years). Thirty-four patients had node-positive disease, and 3 had micrometastasis. No patient in the study population had a stage higher than IIB.

\section{Clinical and pathological characteristics associated with TIL levels}

Tumors with higher nuclear and histologic grade had high TIL levels $(p<0.001$ and $p=0.001$, respectively). Other characteristics, including tumor burden, PR expression, and Ki-67 expression, were not associated with TIL levels.

\section{Correlation between continuous RS and continuous TIL levels}

Pearson's $\mathrm{R}$ test was performed to explore the relationship between continuous RS and continuous TIL levels. A weak but significant correlation was observed between the two continuous parameters (Pearson's $R=0.201 ; p=0.004$; Fig. 1 ).

\section{Comparisons categorized RS and categorized TIL levels}

Of the 97 patients with low-TIL tumors, $56(57.7 \%)$ had a low-RS tumor, 30 (30.9\%) had an intermediate-RS tumor, and 11 (11.3\%) had a high-RS tumor (Fig. 2a). Eighty-eight patients with intermediate-TIL tumors showed a similar distribution of categorized RS compared to patients with low-TIL tumors (Fig. 2a). In contrast, among the patients with high-TIL tumors, the percentage of intermediate RS was $76.9 \%$, which was significantly higher than that in patients with low- or intermediate-TIL tumors $(p=0.007)$. When we compared TIL levels with dichotomized RS, tumors with high TIL levels tended to have higher RS ( $\geq 26$ ), but it was not significant (Fig. 2b; $p=0.155$ ).

Next, we compared RS distributions according to categorized TIL levels because both markers were not normally distributed $(p<0.001$ with Kolmogorov-Smirnov test). The RS distributions differed significantly according to TIL levels $(p<0.001$ with Kruskal-Wallis test; Fig. 3a). A post-hoc test using the Bonferroni-corrected Dunn's procedure showed that high-TIL tumors had significantly higher median RS than low- or intermediate TIL tumors ( $p=0.001$ and $p=0.019$, respectively; Fig. 3a). However, the RS medians did not differ significantly between lowand intermediate-TIL tumors $(p=0.290$, Fig. 3a).

When TIL levels were divided into two groups (lowor intermediate-TILs versus high TILs), high-TIL tumors had significantly higher median RS values than low- or intermediate-TIL tumors (Fig. 3b, $p<0.001$ with MannWhitney U test).

\section{Logistic regression analysis to identify high TIL levels}

We tried to identify factors associated with high TIL levels in ER-positive/HER2-negative tumors. Variables with $p<0.05$ on univariate analysis, including nuclear grade, histologic grade, and RS, were entered as input variables in multivariate analysis in order to distinguish factors associated with high TIL levels. Multivariate analysis revealed that nuclear grade remained the only independent variable associated with high TIL level (Table 2). Nuclear grade demonstrated the highest odds ratio $(\mathrm{OR}=15.025 ; 95 \%$ confidence interval $=2.839$ 79.510) for predicting high TIL levels on the multivariate analysis. 
Table 1 Baseline characteristics

\begin{tabular}{|c|c|c|c|c|}
\hline & Low TIL $(N=97)$ & Intermediate TIL $(N=88)$ & High TIL $(N=13)$ & P-value ${ }^{a}$ \\
\hline Age, median (range) & $50(28-75)$ & $48(34-74)$ & $50(27-73)$ & $0.280^{\mathbf{b}}$ \\
\hline Histology & & & & 0.152 \\
\hline IDC & $73(75.3)$ & $75(85.2)$ & $13(100.0)$ & \\
\hline ILC & $14(14.4)$ & $6(6.8)$ & $0(0.0)$ & \\
\hline Others & $10(10.3)$ & $7(8.0)$ & $0(0.0)$ & \\
\hline pT stage & & & & 0.540 \\
\hline $\mathrm{T} 1$ & $65(67.0)$ & $61(69.3)$ & $7(53.8)$ & \\
\hline $\mathrm{T} 2$ & $32(33.0)$ & $27(30.7)$ & $6(46.2)$ & \\
\hline pN stage & & & & 0.286 \\
\hline No & $84(86.6)$ & $70(79.5)$ & $10(76.9)$ & \\
\hline $\mathrm{N} 1 \mathrm{mi}$ & $5(5.2)$ & $3(3.4)$ & $0(0.0)$ & \\
\hline N1 & $8(8.2)$ & $15(17.0)$ & $3(23.1)$ & \\
\hline pStage & & & & 0.210 \\
\hline IA & $55(56.7)$ & $50(56.8)$ & $6(46.2)$ & \\
\hline IB & $4(4.2)$ & $2(2.3)$ & $0(0.0)$ & \\
\hline$\| \mathrm{A}$ & $37(38.1)$ & $30(34.1)$ & $5(38.5)$ & \\
\hline$\| B$ & $1(1.0)$ & $6(6.8)$ & $2(15.4)$ & \\
\hline Nuclear grade ${ }^{c}$ & & & & $<0.001$ \\
\hline 1 & $4(4.1)$ & $5(5.7)$ & $1(7.7)$ & \\
\hline 2 & 79 (81.5) & $56(63.6)$ & $1(7.7)$ & \\
\hline 3 & $12(12.4)$ & $27(30.7)$ & $11(84.6)$ & \\
\hline Unknown & $2(2.0)$ & $0(0)$ & $0(0)$ & \\
\hline Histologic grade ${ }^{c}$ & & & & 0.001 \\
\hline I & 15 (15.5) & $21(23.9)$ & $0(0)$ & \\
\hline$\|$ & $71(73.2)$ & $57(64.8)$ & $7(53.8)$ & \\
\hline III & $9(9.3)$ & $10(11.4)$ & $6(46.2)$ & \\
\hline Unknown & $2(2.0)$ & $0(0)$ & $0(0)$ & \\
\hline$P R^{d}$ & & & & 0.649 \\
\hline Positive & $81(83.5)$ & 76 (86.4) & $10(76.9)$ & \\
\hline Negative & $16(16.5)$ & 12 (13.6) & $3(23.1)$ & \\
\hline $\mathrm{Ki}-67^{\mathrm{c}}$ & & & & 0.506 \\
\hline$\geq 20 \%$ & 18 (18.6) & $15(17.1)$ & $4(30.8)$ & \\
\hline$<20 \%$ & 79 (81.4) & 72 (81.8) & $9(69.2)$ & \\
\hline Unknown & $0(0.0)$ & $1(1.1)$ & $0(0.0)$ & \\
\hline
\end{tabular}

${ }^{a}{ }^{2}$ test except ${ }^{b}$

${ }^{\mathrm{b}}$ Kruskal-Wallis test

${ }^{c}$ Missing value

dPositive, Allred score 2-8; Negative, Allred score 0-1

Data are presented as $\mathrm{n}(\%)$

IDC: invasive ductal carcinoma, ILC: invasive lobular carcinoma, $p N$ stage: pathologic nodal stage, PR: progesterone receptor, $p S t a g e:$ pathologic stage, $p T$ stage: pathologic tumor stage, TIL: tumor infiltrating lymphocyte

Association of TIL levels with RS-guided adjuvant therapy Lastly, we investigated an association of TIL levels with adjuvant treatment. Based on the RS results, patients were guided to receive either endocrine therapy (ET) alone or chemo-endocrine therapy. As a result, all patients with low RS $(n=98)$ received ET alone, while all patients with high RS $(n=20)$ received chemo-endocrine therapy (Fig. 4a). Among 80 patients with intermediate RS, 45 (56.3\%) were guided to forego ET alone.

Among patients receiving RS-guided treatments, associations between TIL levels and adjuvant treatments were analyzed. The classification by TIL levels was 


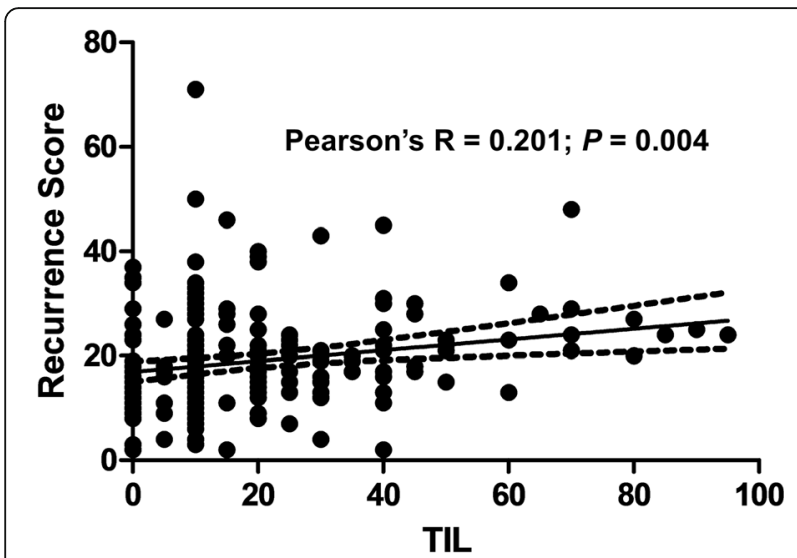

Fig. 1 Scatter plots of continuous recurrence scores and continuous tumor infiltrating lymphocytes (TILs) (Pearson's $R=0.201 ; P=0.004$ )

associated with a significant change in the chemoendocrine treatment rate $(p<0.001$, Fig. $4 \mathrm{~b})$. Chemoendocrine therapy was more frequently performed among patients with high TILs (chemo-endocrine therapy in 10 [76.9\%] and ET alone in 3 [23.1\%]) and less frequently among those with low TILs (chemo-endocrine therapy in 24 [27.3\%] and ET alone in 64 [72.7\%]) or intermediate (chemo-endocrine therapy in 21 [21.6\%] and ET alone in 76 [78.4\%]).

\section{Discussion}

Many investigators have extensively studied the clinical value of TILs or RS in breast cancer; however, there are only a few studies regarding an association between Oncotype DX-RS and TILs in a specific subset of breast cancer, ER-positive/HER2-negative breast cancer.
We previously hypothesized that tumors with extensive lymphocytic infiltration might have high RS because patients with high TIL levels have a higher rate of $\mathrm{pCR}$ to preoperative chemotherapy. Additionally, ER-positive/ HER2-negative patients with high RS derive clinical benefit from adjuvant chemotherapy. If a positive correlation could be found between the two markers, further studies testing TIL levels as a biomarker that predicts clinical benefit for adjuvant chemotherapy in ERpositive/HER2-negative cancer would be warranted.

In this study, we found a weak correlation between RS and TIL levels, wherein tumors with high TIL levels tended to have a higher RS. Moreover, high-TIL tumors had significantly higher median RS than low- or intermediate TIL tumors. A weak correlation between the two continuous markers was also observed. However, a close association between categorical RS and TIL levels was not observed. In addition, multivariate analysis revealed that RS was not an independent factor associated with high TIL levels.

These biomarkers are both associated with aggressive tumor biology, but they depend on different pathways in tumor progression. RS mainly reflects the biological characteristics of tumor cells, while TILs are a type of immune cells, reflecting the immune tumor microenvironment. Therefore, it is not surprising that RS was not highly correlated with TIL levels in our analysis.

On examining 21 genes in RS, the CD68 is the only gene obviously associated with immune function in breast cancer. CD68 is a heavily glycosylated glycoprotein that is highly expressed by macrophages and is recognized as a marker for tumor-associated macrophages [21]. In a hypothesis-generating study using human
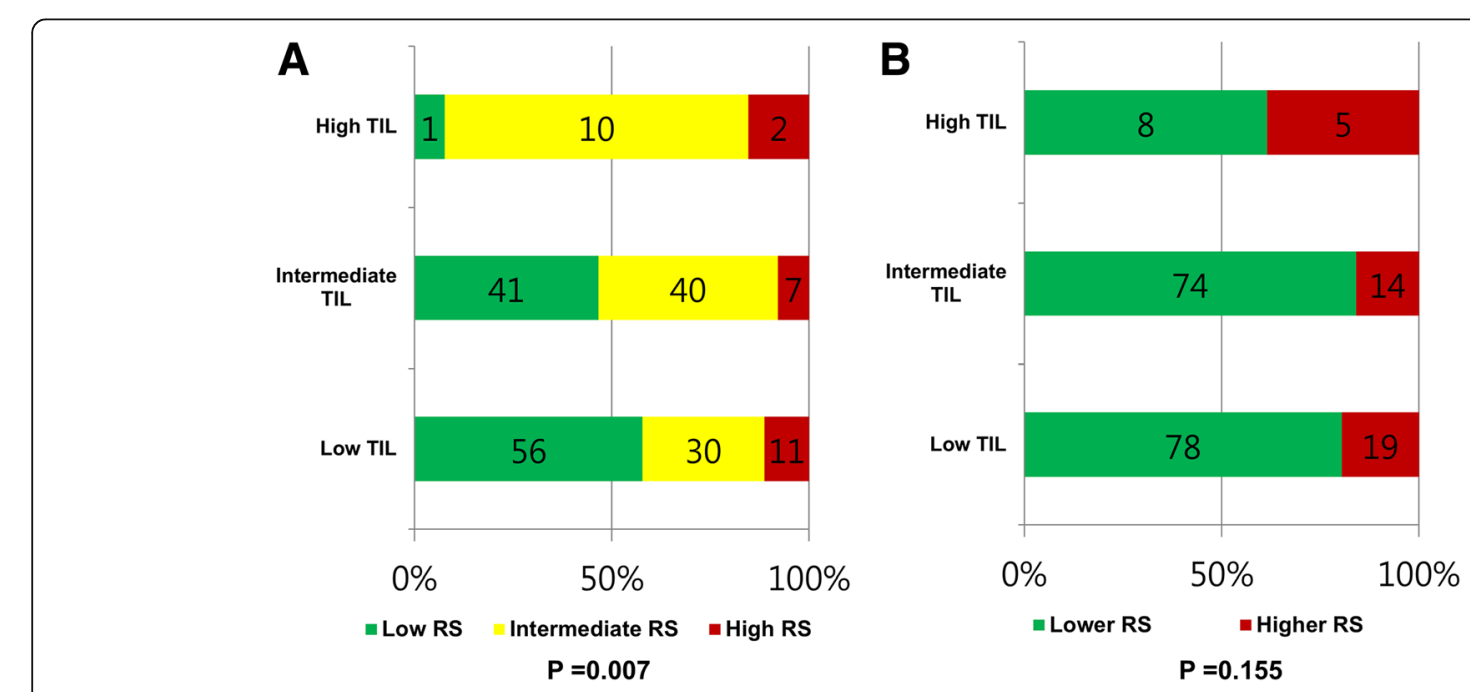

Fig. 2 Distribution of the 21-gene recurrence score (RS) groups according to tumor infiltrating lymphocyte (TIL) count. a Patients with intermediate-TIL tumors showed a distribution of categorized RS similar to that of patients with low-TIL tumors. In contrast, among the patients with high-TIL tumors, the percentage of intermediate RS was significantly higher $(p=0.007)$. $\mathbf{b}$ Tumors with higher RS $(\geq 26)$ tended to have a higher rate of high TIL levels, but it was not significant $(p=0.155)$ 

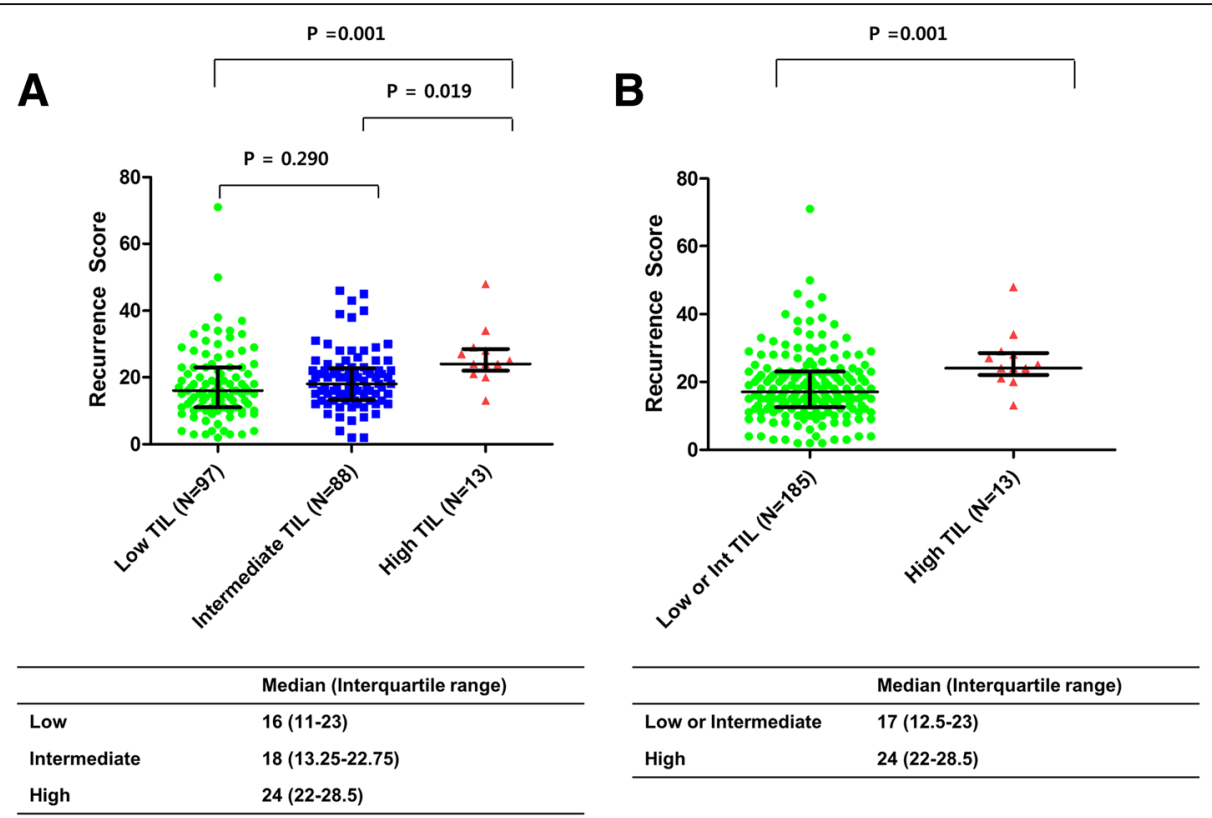

Fig. 3 Distributions of recurrence score (RS) according to tumor infiltrating lymphocyte (TIL) levels. a Distributions of RS differed significantly among the three groups, which were categorized by TIL levels ( $p=0.001$ with Kruskal-Wallis test). The median RS (interquartile) was 16 (11-23) in the low-TIL group, 18 (13.25-22.75) in the intermediate-TIL group, and 24 (22-28.5) in the high-TIL group. A post-hoc test with the Bonferronicorrected Dunn's procedure showed that high-TIL tumors had significantly higher median RS than low- or intermediate TIL tumors ( $p=0.001$ and $p=0.019$, respectively), whereas intermediate-TIL tumors did not have a higher median RS than low-TIL tumors $(p=0.290)$. b Distributions of RS differed significantly according to the dichotomized TIL levels ( $p<0.001$ with Mann-Whitney $U$ test). The median RS was 17 (12.5-23) in the low- and intermediate-TIL group and 24 (22-28.5) in the high-TIL group. Lines indicate the median values, and error bars indicate interquartile ranges

breast carcinoma treated with or without neoadjuvant chemotherapy, CD68 was found to play a role in the immune microenvironment of breast cancer [21]. Specifically, several studies using large cohorts of breast cancer patients have revealed that the expression of CD68 in tumor tissue is associated with higher grade [22, 23], increased angiogenesis [24-26], and reduced disease-free survival $[25,27,28]$. The impact of CD68 expression on the final 21-gene RS has not been of much interest to researchers. In our study, the relationship between TILs and CD68 levels remained unexplored. Hence, further studies correlating CD68 and TIL levels in ER-positive/ HER2-negative cancer will shed light on the tumorimmune interaction in this subset.

There might be an association between HER2 expression and TIL levels because there is solid evidence-from translational studies using tumor samples from prospective trials - that high TIL levels correlate with response to anti-HER2 therapy [29] and that patients with HER2positive breast cancer receiving adjuvant trastuzumab

Table 2 Binary logistic regression analysis to identify predictive factors for high TIL level

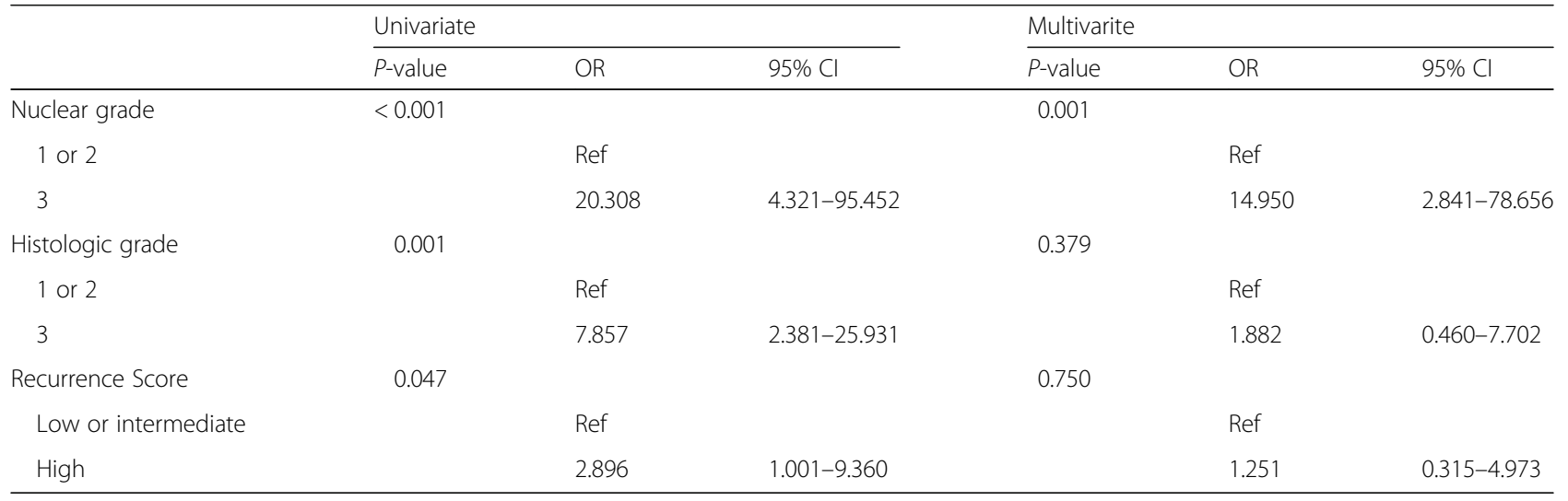

TIL: tumor infiltrating lymphocyte, OR: odds ratio, Cl: confidence interval 

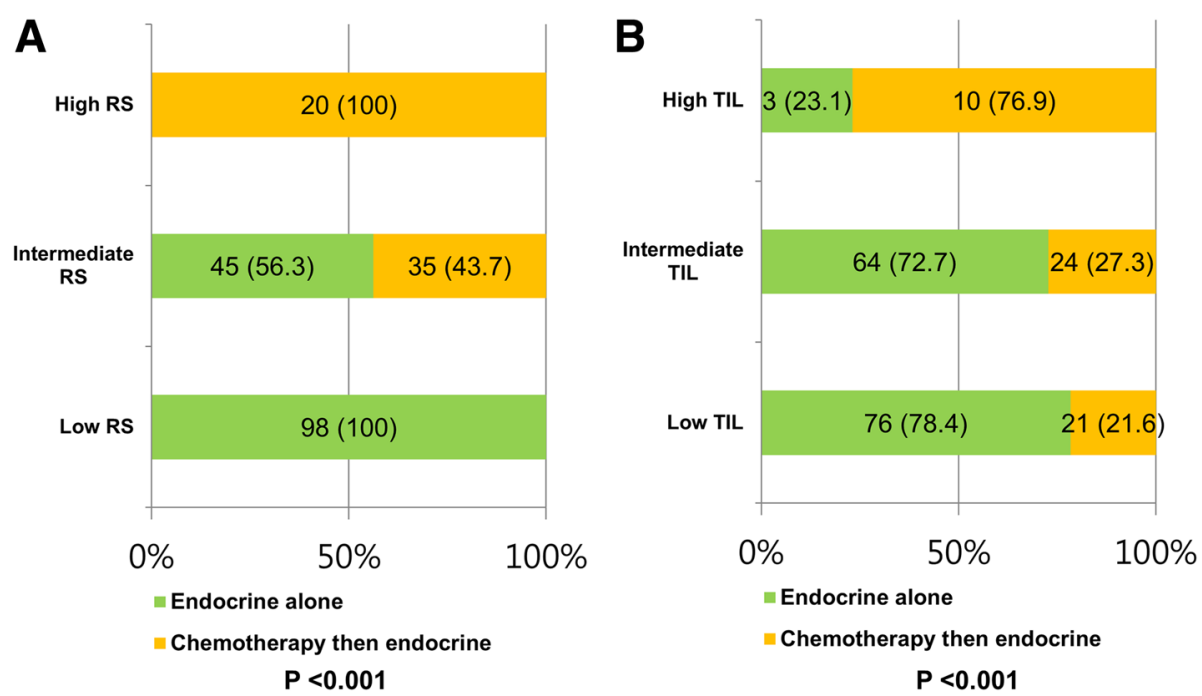

Fig. 4 Association of TIL levels with RS-guided adjuvant therapy. a The proportions of adjuvant treatments according to RS $(p<0.001)$. $\mathbf{b}$ The proportions of adjuvant treatments according to TIL levels $(p<0.001)$

showed improved survival [30]. However, similar to real practice, we only used 21-gene RS for HER2-negative cases; hence, it might be difficult to find a link between TIL levels and HER2 expression in this study.

A growing body of evidence suggests that TIL levels are associated with the response to chemotherapy via several distinct mechanisms of the tumor-immune interaction [2-8]. Chemotherapy can increase the vulnerability of tumor cells to lytic action by cytotoxic CD8+ T cells, and selectively reduce circulating regulatory $\mathrm{T}$ cells as well as restore the function of natural killer (NK) cells [31]. Furthermore, the proliferation of $\mathrm{T}$ cells and the lytic function of NK cells were promoted by chemotherapy in breast cancer patients treated with adjuvant taxanes [32]. These findings are connected with recent studies showing that patients with lymphocyte-predominant breast cancer (LPBC) had a better prognosis compared to non-LPBC among patients treated with chemotherapy $[2,4,6]$. However, the association between TIL levels and oncologic outcome was reproducibly confirmed in TNBC, but not in ER-positive/HER2-negative breast cancer $[2,4,6]$.

A meta-analysis with 1366 HR-positive/HER2-negative patients receiving NAC found that patients with high TIL levels had a worse prognosis than patients with low TIL levels, but a similar outcome compared to patients with intermediate TIL levels, even though they had a higher rate of pCR [10]. By contrast, patients with high RS had worse outcome compared to patients with low or intermediate RS [11, 12]. Taken together with our data, it appears that RS and TIL levels stratify patients into subgroups based on different tumor biology. TIL levels are relatively low in ER-positive/HER2-negative tumors than in other subtypes. Although inconsistent cut-off points $(50 \%$ or $60 \%$ ) have been applied to define high TIL levels, the rate of high-TIL tumors is $2.9 \%-13 \%$ in ER-positive/HER2-negative patients [6, 10]. Our data are in line with these findings, with a rate of high-TIL tumors of only $6.6 \%$ ( 13 of 198 ; cut-off of $60 \%$ ).

The relationship between endocrine responsiveness and the degree of TILs needs to be explored. Recently, Dieci et al. evaluated the association between TIL levels in core biopsies and Ki-67 suppression after letrozole treatment with/without lapatinib for 24 weeks [33]. They found that patients with high TIL levels (cut-off of 10\%) more frequently had a relative Ki-67 suppression $\geq 50 \%$ from baseline compared to patients with low TIL levels, without statistical significance (55\% vs. $35 \%$ ). Dunbier et al. showed that an inflammatory signature is associated with a poor response to 2-week aromatase inhibitor treatment, implying that TIL levels might be associated with endocrine resistance [34]. Further clinical and preclinical research should be performed.

Previously, Krishnamurti et al. compared TIL levels with RS in ER-positive cancer [35], and their study shows several differences from ours. Krishnamurti et al. did not exclude HER2-positive tumors from ER-positive tumors, whereas we only included ER-positive/HER2negative tumors because the RS assay is applied clinically for those specific tumors. Moreover, the TIL level cut-offs are different between the studies. Krishnamurti et al. set the high TIL cut-off of as $50 \%$, whereas we set ours at $60 \%$, per the study by Denkert [10]. In addition, Krishnamurti et al. showed a negative correlation between TIL levels and RS, implying that TIL is a favorable marker that differs from our study. Further studies with larger cohorts are required to determine the prognostic effects of TIL levels in ER-positive/HER2negative breast cancer. 
A major limitation of our study, in addition to the short follow-up duration, is the absence of survival analyses among the groups divided by the two markers. The clinical outcomes of our study population might help refine prognostic discrimination according to these markers. Additionally, evaluation of TIL levels could differ according to the type of sample, such as core biopsies or surgical specimens. In this study, we evaluated the TIL levels using only surgically resected samples, but several studies used samples from core biopsies. Despite these limitations, our analysis comparing RS and TIL levels lays the groundwork for future research on the tumor-immune interaction in ER-positive/HER2-negative breast cancer.

Intriguingly, in our patients receiving RS-guided treatments, chemotherapy was more frequently performed among patients with high TILs and less frequently among those with low or intermediate TILs. Further studies are required considering the clinical outcomes to determine whether TIL levels could clinically benefit chemotherapy for endocrine-sensitive tumors.

\section{Conclusions}

Despite of a very weak correlation between continuous TIL levels and RS, we found that tumors with high TIL levels tended to have a higher RS in ER-positive/HER2negative breast cancer. Further study is warranted considering the clinical outcomes. The role of the tumorimmune interaction in ER-positive breast cancer needs to be explored in the future studies.

\section{Abbreviations}

ANOVA: Analysis of variation; ER: Estrogen receptor; HER2: human epidermal growth factor receptor 2; HR: Hormone receptor; NAC: Neoadjuvant chemotherapy; pCR: Pathologic complete response; PR: Progesterone receptor; RS: Recurrence score; TILs: Tumor-infiltrating lymphocytes; TNBC: Triple-negative breast cancer

\section{Acknowledgments}

The authors thank Ph.D. Hye Sun Lee, Biostatistician, Biostatistics Collaboration Unit, Yonsei University College of Medicine, Seoul, Korea, for her help with statistical analyses.

\section{Funding}

This research was supported by the Basic Science Research Program through the NRF, funded by the Ministry of Science, ICT, \& Future Planning (NRF2015R1C1A1A02037104), and a grant from the National R\&D Program for Cancer Control, Ministry of Health \& Welfare, Republic of Korea (1520120).

\section{Availability of data and materials}

The datasets generated during and/or analyzed during the current study are available from the corresponding author on request.

\section{Authors' contributions}

SGA, YJC and JJ were involved in the study concept and design. Data acquisition was undertaken by SGA, YJC, SJB, CY and HWL. Analysis and interpretation of data were performed by SGA, YJC, SJB, CY, HWL and JJ. SGA and YJC drafted the manuscript. All of the authors have read and approved the final manuscript.

\section{Ethics approval and consent to participate}

The study protocol was reviewed and approved by the Institutional Review Boards of he Gangnam Severance Hospital, Yonsei University, Seoul, Korea, and adhered to the tenets of the Declaration of Helsinki. Owing to retrospective approach of this study, the need for informed consent was waived by the ethics committees.

\section{Consent for publication}

Not applicable.

\section{Competing interests}

The authors declare that they have no competing interest.

\section{Publisher's Note}

Springer Nature remains neutral with regard to jurisdictional claims in published maps and institutional affiliations.

\section{Author details}

${ }^{1}$ Department of Surgery, Gangnam Severance Hospital, Yonsei University College of Medicine, Seoul, Korea. ${ }^{2}$ Department of Pathology, Gangnam Severance Hospital, Yonsei University College of Medicine, Seoul, Korea.

Received: 20 October 2017 Accepted: 15 March 2018

Published online: 24 March 2018

\section{References}

1. Salgado R, Denkert C, Demaria S, Sirtaine N, Klauschen F, Pruneri G, Wienert S, Van den Eynden G, Baehner FL, Penault-Llorca F, et al. The evaluation of tumor-infiltrating lymphocytes (TILs) in breast cancer: recommendations by an international TILs working group 2014. Annals of oncology : official journal of the European Society for Medical Oncology. 2015;26(2):259-71.

2. Adams S, Gray RJ, Demaria S, Goldstein L, Perez EA, Shulman LN, Martino S, Wang $M$, Jones VE, Saphner TJ, et al. Prognostic value of tumor-infiltrating lymphocytes in triple-negative breast cancers from two phase III randomized adjuvant breast cancer trials: ECOG 2197 and ECOG 1199. Journal of clinical oncology : official journal of the American Society of Clinical Oncology. 2014:32(27):2959-66.

3. Denkert C, Loibl S, Noske A, Roller M, Muller BM, Komor M, Budczies J, DarbEsfahani S, Kronenwett R, Hanusch C, et al. Tumor-associated lymphocytes as an independent predictor of response to neoadjuvant chemotherapy in breast cancer. Journal of clinical oncology : official journal of the American Society of Clinical Oncology. 2010;28(1):105-13.

4. Denkert C, von Minckwitz G, Brase JC, Sinn BV, Gade S, Kronenwett R, Pfitzner BM, Salat C, Loi S, Schmitt WD, et al. Tumor-infiltrating lymphocytes and response to neoadjuvant chemotherapy with or without carboplatin in human epidermal growth factor receptor 2-positive and triple-negative primary breast cancers. Journal of clinical oncology : official journal of the American Society of Clinical Oncology. 2015;33(9):983-91.

5. Lee HJ, Seo JY, Ahn JH, Ahn SH, Gong G. Tumor-associated lymphocytes predict response to neoadjuvant chemotherapy in breast cancer patients. J Breast Cancer. 2013;16(1):32-9.

6. Loi S, Sirtaine N, Piette F, Salgado R, Viale G, Van Eenoo F, Rouas G, Francis $P$, Crown JP, Hitre $E$, et al. Prognostic and predictive value of tumorinfiltrating lymphocytes in a phase III randomized adjuvant breast cancer trial in node-positive breast cancer comparing the addition of docetaxel to doxorubicin with doxorubicin-based chemotherapy: BIG 02-98. Journal of clinical oncology : official journal of the American Society of Clinical Oncology. 2013;31(7):860-7.

7. Seo AN, Lee HJ, Kim EJ, Kim HJ Jang MH, Lee HE, Kim YJ, Kim JH, Park SY. Tumour-infiltrating CD8+ lymphocytes as an independent predictive factor for pathological complete response to primary systemic therapy in breast cancer. Br J Cancer. 2013;109(10):2705-13.

8. West NR, Kost SE, Martin SD, Milne K, Deleeuw RJ, Nelson BH, Watson PH. Tumour-infiltrating FOXP3(+) lymphocytes are associated with cytotoxic immune responses and good clinical outcome in oestrogen receptornegative breast cancer. Br J Cancer. 2013;108(1):155-62.

9. Issa-Nummer Y, Darb-Esfahani S, Loibl S, Kunz G, Nekljudova V, Schrader I, Sinn BV, Ulmer HU, Kronenwett R, Just M, et al. Prospective validation of immunological infiltrate for prediction of response to neoadjuvant chemotherapy in HER2-negative breast cancer-a substudy of the neoadjuvant GeparQuinto trial. PLoS One. 2013;8(12):e79775. 
10. Denkert C, von Minckwitz G, Darb-Esfahani S, Lederer B, Heppner BI, Weber KE, Budczies J, Huober J, Klauschen F, Furlanetto J, et al. Tumour-infiltrating lymphocytes and prognosis in different subtypes of breast cancer: a pooled analysis of 3771 patients treated with neoadjuvant therapy. The Lancet Oncology. 2018;19(1):40-50.

11. Paik S, Shak S, Tang G, Kim C, Baker J, Cronin M, Baehner FL, Walker MG, Watson D, Park T, et al. A multigene assay to predict recurrence of tamoxifen-treated, node-negative breast cancer. N Engl J Med. 2004;351(27): 2817-26.

12. Paik S, Tang G, Shak S, Kim C, Baker J, Kim W, Cronin M, Baehner FL, Watson $D$, Bryant J, et al. Gene expression and benefit of chemotherapy in women with node-negative, estrogen receptor-positive breast cancer. Journal of clinical oncology : official journal of the American Society of Clinical Oncology. 2006;24(23):3726-34.

13. Gianni L, Zambetti M, Clark K, Baker J, Cronin M, Wu J, Mariani G, Rodriguez J, Carcangiu M, Watson D, et al. Gene expression profiles in paraffinembedded core biopsy tissue predict response to chemotherapy in women with locally advanced breast cancer. Journal of clinical oncology : official journal of the American Society of Clinical Oncology. 2005;23(29):7265-77.

14. Yardley DA, Peacock NW, Shastry M, Burris HA 3rd, Bechhold RG, Hendricks CB, Yoshizawa CN, Sing AP, Hainsworth JD. A phase II trial of ixabepilone and cyclophosphamide as neoadjuvant therapy for patients with HER2negative breast cancer: correlation of pathologic complete response with the 21-gene recurrence score. Breast Cancer Res Treat. 2015;154(2):299-308.

15. Ahn SG, Lee HM, Cho SH, Bae SJ, Lee SA, Hwang SH, Jeong J, Lee HD. The difference in prognostic factors between early recurrence and late recurrence in estrogen receptor-positive breast cancer: nodal stage differently impacts early and late recurrence. PLoS One. 2013;8(5):e63510.

16. Elston CW, Ellis IO. Pathological prognostic factors in breast cancer. I. The value of histological grade in breast cancer: experience from a large study with long-term follow-up. Histopathology. 1991;19(5):403-10.

17. Harvey JM, Clark GM, Osborne CK, Allred DC. Estrogen receptor status by immunohistochemistry is superior to the ligand-binding assay for predicting response to adjuvant endocrine therapy in breast cancer. Journal of clinical oncology : official journal of the American Society of Clinical Oncology. 1999;17(5):1474-81.

18. Ahn SG, Lee JH, Lee HW, Jeon TJ, Ryu YH, Kim KM, Sohn J, Yun M, Lee SA, Jeong J, et al. Comparison of standardized uptake value of 18F-FDG-PET-CT with 21-gene recurrence score in estrogen receptor-positive, HER2-negative breast cancer. PLoS One. 2017:12(4):e0175048.

19. Wolff AC, Hammond ME, Hicks DG, Dowsett M, McShane LM, Allison KH, Allred DC, Bartlett JM, Bilous M, Fitzgibbons P, et al. Recommendations for human epidermal growth factor receptor 2 testing in breast cancer: American Society of Clinical Oncology/College of American Pathologists clinical practice guideline update. Journal of clinical oncology : official journal of the American Society of Clinical Oncology. 2013;31(31):3997-4013.

20. Sparano JA, Gray RJ, Makower DF, Pritchard KI, Albain KS, Hayes DF, Geyer CE $\mathrm{Jr}$, Dees EC, Perez EA, Olson JA Jr, et al. Prospective validation of a 21-gene expression assay in breast Cancer. N Engl J Med. 2015;373(21):2005-14.

21. Ruffell B, Au A, Rugo HS, Esserman L, Hwang ES, Coussens LM. Leukocyte composition of human breast cancer. Proc Natl Acad Sci U S A. 2012;109(8): 2796-801.

22. Lee AH, Happerfield LC, Bobrow LG, Millis RR. Angiogenesis and inflammation in invasive carcinoma of the breast. J Clin Pathol. 1997;50(8):669-73.

23. Esserman $L$, Kumar AS, Herrera AF, Leung J, Au A, Chen YY, Moore DH, Chen DF, Hellawell J, Wolverton D, et al. Magnetic resonance imaging captures the biology of ductal carcinoma in situ. Journal of clinical oncology : official journal of the American Society of Clinical Oncology. 2006;24(28):4603-10

24. Uzzan B, Nicolas P, Cucherat M, Perret GY. Microvessel density as a prognostic factor in women with breast cancer: a systematic review of the literature and meta-analysis. Cancer Res. 2004;64(9):2941-55.

25. Tsutsui S, Yasuda K, Suzuki K, Tahara K, Higashi H, Era S. Macrophage infiltration and its prognostic implications in breast cancer: the relationship with VEGF expression and microvessel density. Oncol Rep. 2005;14(2):425-31.

26. Bolat F, Kayaselcuk F, Nursal TZ, Yagmurdur MC, Bal N, Demirhan B. Microvessel density, VEGF expression, and tumor-associated macrophages in breast tumors: correlations with prognostic parameters. Journal of experimental \& clinical cancer research : CR. 2006;25(3):365-72.
27. Leek RD, Lewis CE, Whitehouse R, Greenall M, Clarke J, Harris AL. Association of macrophage infiltration with angiogenesis and prognosis in invasive breast carcinoma. Cancer Res. 1996;56(20):4625-9.

28. Campbell MJ, Tonlaar NY, Garwood ER, Huo D, Moore DH, Khramtsov Al, Au A, Baehner F, Chen Y, Malaka DO, et al. proliferating macrophages associated with high grade, hormone receptor negative breast cancer and poor clinical outcome. Breast Cancer Res Treat. 2011;128(3):703-11.

29. Salgado R, Denkert C, Campbell C, Savas P, Nuciforo P, Aura C, de Azambuja E, Eidtmann H, Ellis CE, Baselga J, et al. Tumor-infiltrating lymphocytes and associations with pathological complete response and event-free survival in HER2-positive early-stage breast Cancer treated with Lapatinib and Trastuzumab: a secondary analysis of the NeoALTTO trial. JAMA oncology. 2015;1(4):448-54.

30. Loi S, Michiels S, Salgado R, Sirtaine N, Jose V, Fumagalli D, KellokumpuLehtinen PL, Bono P, Kataja V, Desmedt C, et al. Tumor infiltrating lymphocytes are prognostic in triple negative breast cancer and predictive for trastuzumab benefit in early breast cancer: results from the FinHER trial. Annals of oncology : official journal of the European Society for Medical Oncology. 2014;25(8):1544-50.

31. van der Most RG, Currie AJ, Cleaver AL, Salmons J, Nowak AK, Mahendran S, Larma I, Prosser A, Robinson BW, Smyth MJ, et al. Cyclophosphamide chemotherapy sensitizes tumor cells to TRAlL-dependent CD8 T cellmediated immune attack resulting in suppression of tumor growth. PLoS One. 2009;4(9):e6982.

32. Carson WE 3rd, Shapiro CL, Crespin TR, Thornton LM, Andersen BL. Cellular immunity in breast cancer patients completing taxane treatment. Clinical cancer research : an official journal of the American Association for Cancer Research. 2004;10(10):3401-9.

33. Dieci MV, Frassoldati A, Generali D, Bisagni G, Piacentini F, Cavanna L, Cagossi K, Puglisi F, Michelotti A, Berardi R, et al. Tumor-infiltrating lymphocytes and molecular response after neoadjuvant therapy for HR +/HER2- breast cancer: results from two prospective trials. Breast Cancer Res Treat. 2017;163(2):295-302

34. Dunbier AK, Ghazoui Z, Anderson H, Salter J, Nerurkar A, Osin P, A'Hern R, Miller WR, Smith IE, Dowsett M. Molecular profiling of aromatase inhibitortreated postmenopausal breast tumors identifies immune-related correlates of resistance. Clinical cancer research : an official journal of the American Association for Cancer Research. 2013;19(10):2775-86.

35. Krishnamurti U, Wetherilt CS, Yang J, Peng L, Li X. Tumor-infiltrating lymphocytes are significantly associated with better overall survival and disease-free survival in triple-negative but not estrogen receptor-positive breast cancers. Hum Pathol. 2017:64:7-12.

\section{Submit your next manuscript to BioMed Central and we will help you at every step:}

- We accept pre-submission inquiries

- Our selector tool helps you to find the most relevant journal

- We provide round the clock customer support

- Convenient online submission

- Thorough peer review

- Inclusion in PubMed and all major indexing services

- Maximum visibility for your research

Submit your manuscript at www.biomedcentral.com/submit
C) Biomed Central 